Bulletin of the Institute of Mathematics

Academia Sinica (New Series)

Vol. 13 (2018), No. 1, pp. 1-29

DOI: 10.21915 /BIMAS.2018101

\title{
ONE- $W$-TYPE MODULES FOR RATIONAL CHEREDNIK ALGEBRA AND CUSPIDAL TWO-SIDED CELLS
}

\author{
DAN CIUBOTARU
}

To George Lusztig, with admiration

Mathematical Institute, University of Oxford, Oxford, OX2 6GG, UK.

E-mail: dan.ciubotaru@maths.ox.ac.uk

\|\|\|\|

\begin{abstract}
We classify the simple modules for the rational Cherednik algebra $\mathbf{H}_{0, c}$ that are irreducible when restricted to $W$, in the case when $W$ is a finite Weyl group. The classification turns out to be closely related to the cuspidal two-sided cells in the sense of Lusztig. We compute the Dirac cohomology of these modules and use the tools of Dirac theory to find nontrivial relations between the cuspidal Calogero-Moser cells, in the sense of Bellamy, and the cuspidal two-sided cells.
\end{abstract}

\section{Introduction}

1.1 In this paper, we classify the simple modules for the rational Cherednik algebra $\mathbf{H}_{0, c}, c$ arbitrary, that are irreducible when restricted to $W$, in the case when $W$ is a finite Weyl group. We call these modules one-W-type modules. We find that such modules exist when $W$ is of type $B_{n}, D_{n}, G_{2}$, $F_{4}, E_{6}$, and $E_{8}$, but not in type $A_{n-1}$ (as expected from [14]) or in type $E_{7}$. The classification result is Theorem 3.5 .

In [12], we introduced a Dirac operator and the notion of Dirac cohomology in the setting of the graded Hecke algebras defined by Drinfeld, extending in this way the construction from [2] for Lusztig's graded affine

Received September 3, 2016 and in revised form September 18, 2016.

AMS Subject Classification: 20C08, 20F55.

Key words and phrases: Rational Cherednik algebra, affine Hecke algebra, cells, Weyl group, Dirac cohomology.

This research was supported in part the EPSRC grant EP/N033922/1. 
Hecke algebras [21]. In particular, the constructions in [12] apply to rational Cherednik algebras. The Dirac cohomology of one- $W$-type modules turns out to be easy to compute and yet it yields a good amount of nontrivial information. The results of this paper can be viewed as the rational Cherednik algebra analogues of the results of [3, 13] concerning one- $W$-type modules for the graded affine Hecke algebra, where the motivation comes from the study of isolated unitary representations of reductive $p$-adic groups.

The main application of this method is that it gives a direct relation between the cuspidal components in the partition of $\operatorname{Irr} W$ coming from considering certain fibers over the Calogero-Moser $(\mathrm{CM})$ space $X_{c}(W)=$ $\operatorname{Spec}\left(\mathbf{H}_{0, c}\right)$ (see for example [14], [19], [18]), and the cuspidal two-sided cells in $\operatorname{Ir} W$, in the sense of Lusztig [20]. The CM partition of $\operatorname{Irr}(W)$ is expected (see [19]) to be related to the one into two-sided cells (or families) of representations, in the sense of Lusztig [20] for real reflection groups, and [24] for complex reflection groups. At least when $W$ is a real reflection group, the conjecture is that the two partitions coincide. This is known to hold in several cases, for example in type $A$ [14], and for the class of complex reflection groups $G(m, d, n)$ by [19], [4], [22], and [7]. See also [9] for more details as well as for a refinement of this conjecture.

The two notions of cuspidal that we refer to in this paper are as follows:

(1) In the case of Lusztig's two-sided cells [20], the cuspidal ones are the building blocks of all cells via truncated induction and tensoring with the sign representation, see Definition 4.4.

(2) In the case of the Calogero-Moser space, let

$$
\Theta: \operatorname{lrr}(W) \rightarrow X_{c}(W)
$$

be the morphism defined by [18], more details are in the body of the paper. The CM cells (or families) are, by definition, the fibers of this map. Following [4], we call a CM cell cuspidal if its image under $\Theta$ is a zero-dimensional leaf of $X_{c}(W)$. (See [10], [4] for the relevant results about the Poisson variety structure of $X_{c}(W)$, particularly [10, Theorem 3].) 
To see that the one-W-type modules are necessarily related to cuspidal CM cells, recall from [4, Theorem 1, Corollary 1] that if $M$ is a simple $H_{c}(W)$ module whose central character is not a cuspidal point in $X_{c}(W)$, then as a $W$-representation $M$ must be parabolically induced from a representation of a proper parabolic subgroup of $W$. Since an irreducible $W$-representation is not proper parabolically induced (section 4.3), we conclude that every irreducible $W$-representation that can be lifted to an $\mathbf{H}_{0, c}$-module belongs to a cuspidal CM cell. For the same reason, the one-W-type modules, under the name rigid modules, were also considered independently by Bellamy and Thiel [7] for the infinite families of complex reflection groups.

1.2. Assume now that the rational Cherednik algebra has equal parameters. In Theorem 4.6, using the approach via the Dirac operator and one- $W$-types, we find nontrivial relations between the cuspidal CM cells and Lusztig's cuspidal families. More precisely, recall that cuspidal families exist only for $B_{n}, n=d^{2}+d, D_{n}, n=d^{2}, G_{2}, F_{4}, E_{6}, E_{7}$, and $E_{8}$, and in each of these cases there exists only one such family, which we denote by $\mathcal{F}_{\text {cusp }}(W)$. For example, when $W=E_{8}$, there are 17 representations in $\mathcal{F}_{\text {cusp }}(W)$.

It turns out, see Theorem 3.5, that $\mathbf{H}_{0,1}$ admits one- $W$-type modules if and only if $W$ has a cuspidal two-sided cell except when $W=E_{7}$, in which case $\mathbf{H}_{0,1}$ does not have one- $W$-type modules. When one- $W$-type modules for $\mathbf{H}_{0,1}$ do exist, they correspond to the same point in $X_{1}(W)$ which we denote $\mathbf{0}$. The point $\mathbf{0}$ must be a zero-dimensional leaf of $X_{1}(W)$ in these cases as explained above. For uniformity of notation, let us denote $\mathbf{0}=\Theta\left(512_{a}^{\prime}\right)$ in the case of $E_{7} . \quad\left(512_{a}^{\prime}\right.$ is one of the two irreducible $W$ representations in the cuspidal two-sided cell of $E_{7}$.)

Theorem 1.1 (also Theorem4.6). Let $W$ be a simple finite Weyl group and let $\mathbf{H}_{0,1}$ be the rational Cherednik algebra with equal parameters. Suppose $W$ has a cuspidal two-sided cell. If $W \neq E_{8}$, then

$$
\mathcal{F}_{\text {cusp }}(W) \subseteq \Theta^{-1}(\mathbf{0})
$$

When $W=E_{8}$, then

$$
\mathcal{F}_{\text {cusp }}\left(E_{8}\right) \backslash\left\{4480_{y}\right\} \subseteq \Theta^{-1}(\mathbf{0}) \text {. }
$$


The notation for $W$-representations is as in [20]. The proof of Theorem 1.1 uses only Theorem [3.5 and elements of Dirac cohomology. While the method of the proof is uniform, certain details, such as decomposition of tensor products, have to be checked case by case. The idea is very simple. The Dirac cohomology theory of these one- $W$-modules implies that if $\sigma$ is an irreducible $W$-representation that can be extended to a one-W-type module of $\mathbf{H}_{0, c}$, then every irreducible constituent of $\sigma \otimes \wedge \mathfrak{h}$ must be in the same CMcell as $\sigma$. The method also applies to unequal parameter cases, and possibly to complex reflection groups as well, but we do not consider those analogues of Theorem 1.1 here beyond Remark 4.8. (The classification of one- $W$-type modules, Theorem 3.5, is obtained for arbitrary unequal parameters though.)

Remark 1.2. The results of Bellamy-Thiel [7] imply that in fact

$$
\mathcal{F}_{\text {cusp }}(W)=\Theta^{-1}(\mathbf{0})
$$

if $W$ is of type $B_{n}$ or $D_{n}$. For exceptional Weyl groups, our methods also give that $\mathcal{F}_{\text {cusp }}(W)=\Theta^{-1}(\mathbf{0})$ for $G_{2}, F_{4}, E_{6}, E_{7}$, but only the weaker statement that

$$
\Theta^{-1}(\mathbf{0}) \subseteq \mathcal{F}_{\text {cusp }}\left(E_{8}\right) \cup\left\{2100_{y}\right\}
$$

for $E_{8}$.

Of course, the expectation is that for $E_{8}$ as well, $\mathcal{F}_{\text {cusp }}(W)=\Theta^{-1}(\mathbf{0})$, but this does not follow from this calculation of one- $W$-types. See Remark 4.7 for more details about this case, in particular for an explanation why the statement $\mathcal{F}_{\text {cusp }}\left(W\left(E_{8}\right)\right)=\Theta^{-1}(\mathbf{0})$ becomes equivalent to proving that $\left\{2100_{y}\right\}$ is a singleton CM-cell.

\section{The Dirac Operator for Rational Cherednik Algebra}

In this section, we recall from [12] the definition of Dirac cohomology for rational Cherednik algebra modules and the basic properties. For the purpose of this paper, we only need to consider the rational Cherednik algebra at $t=0$. 


\subsection{Rational Cherednik algebra at $t=0$}

As before, let $\mathfrak{h}$ be a dimensional $\mathbb{C}$-vector space, denote by $\mathfrak{h}^{*}$ its dual, and $V=\mathfrak{h}+\mathfrak{h}^{*}$. Let $\langle\rangle:, V \times V \rightarrow \mathbb{C}$ be the bilinear symmetric pairing defined by

$$
\langle x, x\rangle=0,\langle y, y\rangle=0,\langle x, y\rangle=\langle y, x\rangle=x(y)
$$

for all $x \in \mathfrak{h}^{*}$ and $y \in \mathfrak{h}$. Let $W \subset G L(\mathfrak{h})$ be a complex reflection group with set of pseudo-reflections $\mathcal{R}$ acting diagonally on $V$. The form $\langle$,$\rangle is$ $W$-invariant.

For every reflection $s \in \mathcal{R}$, the spaces $\left.\operatorname{im}\left(\operatorname{Id}_{V}-s\right)\right|_{\mathfrak{h}^{*}}$ and $\left.\operatorname{im}\left(\operatorname{Id}_{V}-s\right)\right|_{\mathfrak{h}}$ are one-dimensional. Choose $\alpha_{s}$ and $\alpha_{s}^{\vee}$ nonzero elements in $\left.\operatorname{im}\left(\operatorname{Id}_{V}-s\right)\right|_{\mathfrak{h}^{*}}$ and $\left.\operatorname{im}\left(\operatorname{Id}_{V}-s\right)\right|_{\mathfrak{h}}$, respectively. Then there exists $\lambda_{s} \in \mathbb{C}, \lambda_{s} \neq 1$ a root of unity, such that

$$
s\left(\alpha_{s}^{\vee}\right)=\lambda_{s} \alpha_{s}^{\vee}, s\left(\alpha_{s}\right)=\lambda_{s}^{-1} \alpha_{s}
$$

(In the case when $W$ is a finite reflection group, $\lambda_{s}=-1$.) For every $v \in V$ such that $\langle v, v\rangle \neq 0$, denote by $s_{v}$ the reflection in the hyperplane perpendicular to $v$. The reflection $s_{v}$ is given by:

$$
s_{v}(u)=u-\frac{2}{\langle v, v\rangle}\langle u, v\rangle v, u \in V .
$$

Let $\sqrt{\lambda_{s}}$ be a square root of $\lambda_{s}$. Notice that $s=s_{v_{s}} s_{v_{s}^{\prime}} \in O(V)$, where $v_{s}=\sqrt{\lambda_{s}} \alpha_{s}^{\vee}+\alpha_{s}$ and $v_{s}^{\prime}=\alpha_{s}^{\vee}+\sqrt{\lambda_{s}} \alpha_{s}$.

Definition 2.1. The rational Cherednik algebra $\mathbf{H}_{0, c}$ associated to $\mathfrak{h}, W$ and the $W$-invariant parameter function $c: \mathcal{R} \rightarrow \mathbb{C}$ is the quotient of $T(V) \rtimes W$ by the relations:

(1) $\left[y_{1}, y_{2}\right]=0,\left[x_{1}, x_{2}\right]=0$, for all $y_{1}, y_{2} \in \mathfrak{h}, x_{1}, x_{2} \in \mathfrak{h}^{*}$;

(2) $[y, x]=-\sum_{s \in \mathcal{R}} c_{s} \frac{\left\langle y, \alpha_{s}\right\rangle\left\langle\alpha_{s}^{\vee}, x\right\rangle}{\left\langle\alpha_{s}^{\vee}, \alpha_{s}\right\rangle} s$, for all $y \in \mathfrak{h}, x \in \mathfrak{h}^{*}$.

Let $\left\{y_{i}\right\}$ be a basis of $\mathfrak{h}$ and $\left\{x_{i}\right\}$ the dual basis of $\mathfrak{h}^{*}$. Define the element

$$
\Omega_{\mathbf{H}}=2 \sum_{i} x_{i} y_{i}-2 \sum_{s \in \mathcal{R}} \frac{c_{s}}{1-\lambda_{s}} s \in Z\left(\mathbf{H}_{0, c}\right) .
$$




\subsection{The Clifford algebra}

Let $C(V)$ be the complex Clifford algebra defined by $V$ and $\langle$,$\rangle . In$ terms of the basis $x_{i}, y_{i}$ 's the relations in $C(V)$ are:

$$
x_{i} \cdot x_{j}=-x_{j} \cdot x_{i}, y_{i} \cdot y_{j}=-y_{j} \cdot y_{i}, x_{i} \cdot y_{j}+y_{j} \cdot x_{i}=-2 \delta_{i, j}
$$

Since $V$ is even dimensional, $C(V)$ has a unique complex simple module $S$. The spin module $S$ is realized on the vector space $\bigwedge \mathfrak{h}$ with the action:

$$
\begin{aligned}
& y \cdot\left(y_{1} \wedge \cdots \wedge y_{k}\right)=y \wedge y_{1} \wedge \cdots \wedge y_{k}, \quad y \in \mathfrak{h} ; \\
& x \cdot\left(y_{1} \wedge \cdots \wedge y_{k}\right)=2 \sum_{i}(-1)^{i}\left\langle y_{i}, x\right\rangle y_{1} \wedge \cdots \wedge \hat{y}_{i} \wedge \cdots \wedge y_{k} .
\end{aligned}
$$

\subsection{Pin cover of $W$}

Following [12, $§ 2.3]$, for every $s \in S$, define

$$
\tau_{s}=\frac{1-\lambda_{s}}{2\left\langle\alpha_{s}^{\vee}, \alpha_{s}\right\rangle} \alpha_{s} \alpha_{s}^{\vee}+1 \in C(V) .
$$

By [12, Lemma 4.6], the map $s \mapsto \tau_{s}$ extends to a group homomorphism

$$
\tau: W \rightarrow C(V)^{\times}
$$

Define $\tau_{w}$ to be the image in $C(V)^{\times}$of $w \in W$ under this map.

Since $C(V)$ acts on $S$, we get an action of $\tau(W)$ on $S$.

Lemma 2.2 ([12, Lemma 4.8]). The action of $\tau(W)$ on $S$ preserves each piece $\Lambda^{\ell} \mathfrak{h}$ of $S$, where it acts by the dual of the natural action, i.e.:

$$
\tau_{s} \cdot\left(y_{1} \wedge \cdots \wedge y_{\ell}\right)=\operatorname{det}_{\mathfrak{h}}(s) s\left(y_{1}\right) \wedge \cdots \wedge s\left(y_{\ell}\right) .
$$

\subsection{The Dirac element}

The Dirac element in $\mathbf{H}_{0, c} \otimes C(V)$ is:

$$
\mathcal{D}=\sum_{i} x_{i} \otimes y_{i}+\sum_{i} y_{i} \otimes x_{i}
$$


We denote by

$$
\Delta: \mathbb{C}[W] \rightarrow \mathbf{H}_{t, c} \otimes C(V)
$$

the linear map that extends the assignment $w \mapsto w \otimes \tau_{w}$.

Proposition 2.3 ([12, Proposition 4.9]). The Dirac element has the following properties in $\mathbf{H}_{0, c} \otimes C(V)$ :

(1) $\mathcal{D}$ is invariant with respect to the conjugation action of $\Delta(W)$.

(2) The square equals:

$$
\mathcal{D}^{2}=-\Omega_{\mathbf{H}} \otimes 1-\Delta\left(\Omega_{W, c}\right),
$$

with $\Omega_{\mathbf{H}} \in Z\left(\mathbf{H}_{0, c}\right)$ given by (2.1.3) and

$$
\Omega_{W, c}=\sum_{s \in \mathcal{R}} \frac{2 c_{s}}{1-\lambda_{s}} s \in \mathbb{C}[W]^{W} .
$$

\subsection{Dirac cohomology}

Let $X$ be a finite dimensional $\mathbf{H}_{0, c}$-module. The Dirac operator of $X$ (and $S$ ) is

$$
D_{X}: X \otimes S \rightarrow X \otimes S
$$

given by the action of the Dirac element $\mathcal{D}$. The Dirac cohomology of $X$ $($ and $\mathcal{S}$ ) is

$$
H_{D}(X)=\operatorname{ker} D_{X} / \operatorname{ker} D_{X} \cap \operatorname{im} D_{X}
$$

If nonzero, $H_{D}(X)$ is a finite dimensional $W$-representation.

The state now the main results about Dirac cohomology applied to this setting. Recall that the center $Z\left(\mathbf{H}_{0, c}\right)$ is nontrivial.

Theorem 2.4 ([12, Theorem 3.5 and Theorem 3.8]). For every $z \in Z\left(\mathbf{H}_{0, c}\right)$ there exists a unique element $\zeta_{0, c}(z) \in \mathbb{C}[W]^{W}$ and an element $a \in\left(\mathbf{H}_{0, c} \otimes\right.$ $C(V))^{W}$ such that

$$
z \otimes 1=\Delta\left(\zeta_{0, c}(z)\right)+\mathcal{D} a+a \mathcal{D} \text { in } \mathbf{H}_{0, c} \otimes C(V)
$$

Moreover, the assignment $\zeta_{0, c}: Z\left(\mathbf{H}_{0, c}\right) \rightarrow \mathbb{C}[W]^{W}$ is an algebra homomorphism. 
Consider the dual morphism

$$
\zeta_{0, c}^{*}: \operatorname{Irr}(W)=\operatorname{Spec} \mathbb{C}[W]^{W} \rightarrow \operatorname{Spec}\left(Z\left(\mathbf{H}_{0, c}\right)=X_{c}(W) .\right.
$$

The space $X_{c}(W)$ in the image of $\zeta_{0, c}^{*}$ is the generalized Calogero-Moser space [14].

Theorem 2.5 ([12, Theorem 3.14]). Let $X$ be a finite dimensional $\mathbf{H}_{0, c^{-}}$ module and assume that $Z\left(\mathbf{H}_{0, c}\right)$ acts on $X$ via the central character $\chi \in$ $\operatorname{Spec}\left(Z\left(\mathbf{H}_{0, c}\right)\right)$. Suppose $H_{D}(X) \neq 0$. If $\sigma \in \operatorname{Irr}(W)$ is such that

$$
\operatorname{Hom}_{W}\left[\sigma, H_{D}(X)\right] \neq 0,
$$

then

$$
\chi=\zeta_{0, c}^{*}(\sigma)
$$

By [14], the center $Z\left(\mathbf{H}_{0, c}\right)$ contains the subalgebra $\mathfrak{m}:=S(\mathfrak{h})^{W} \otimes$ $S\left(\mathfrak{h}^{*}\right)^{W}$ and it is a free $\mathfrak{m}$-module of rank $|W|$. The inclusion $\mathfrak{m} \subset Z\left(\mathbf{H}_{0, c}\right)$ induces a surjective morphism

$$
\Upsilon: X_{c}(W) \rightarrow \mathfrak{h}^{*} / W \times \mathfrak{h} / W
$$

Let $\mathfrak{m}_{+}$be the augmentation ideal of $\mathfrak{m}$ and define similarly $S(\mathfrak{h})_{+}^{W}$ and $S\left(\mathfrak{h}^{*}\right)_{+}^{W}$. Then [12, Theorem 5.8] says that the algebra homomorphism from Theorem 2.4 factors through $Z\left(\mathbf{H}_{0, c}\right) / \mathfrak{m}_{+}$:

$$
\zeta_{0, c}: Z\left(\mathbf{H}_{0, c}\right) / \mathfrak{m}_{+} \rightarrow \mathbb{C}[W]^{W},
$$

and so the dual morphism is

$$
\zeta_{0, c}^{*}: \operatorname{Irr}(W) \rightarrow \Upsilon^{-1}(0)
$$

Following [18], let us consider the "baby Verma modules" for $\mathbf{H}_{0, c}$. Define

$$
\overline{\mathbf{H}}_{0, c}=\mathbf{H}_{0, c} / \mathfrak{m}_{+} \mathbf{H}_{0, c}
$$

This is a finite dimensional algebra of dimension $|W|^{3}$, isomorphic to $S\left(\mathfrak{h}^{*}\right)_{W}$ $\otimes S(\mathfrak{h})_{W} \otimes \mathbb{C}[W]$ as a vector space, where we denote by $S(\mathfrak{h})_{W}=S(\mathfrak{h}) / S(\mathfrak{h})_{+}^{W}$ the graded algebra of coinvariants and similarly for $S\left(\mathfrak{h}^{*}\right)_{W}$. 
For every $\left(\sigma, V_{\sigma}\right) \in \operatorname{Irr}(W)$, let

$$
\bar{M}(\sigma)=\overline{\mathbf{H}}_{0, c} \otimes_{S(\mathfrak{h})_{W} \rtimes \mathbb{C}[W]} V_{\sigma}
$$

be the baby Verma module induced from $\sigma$. Here $S(\mathfrak{h})_{W}$ acts by 0 on $V_{\sigma}$.

Theorem 2.6 ([18, Proposition 4.3]).

(1) For every $\sigma \in \operatorname{Irr}(W)$, the module $\bar{M}(\sigma)$ is indecomposable and it has a unique simple quotient $\bar{L}(\sigma)$.

(2) The set $\{\bar{L}(\sigma): \sigma \in \operatorname{Irr}(W)\}$ gives a complete list of non isomorphic simple $\overline{\mathbf{H}}_{0, c}$-modules.

As a consequence $([18, \S 5.4])$, the map

$$
\Theta: \operatorname{Irr}(W) \rightarrow \Upsilon^{-1}(0)=\operatorname{Spec} Z\left(\overline{\mathbf{H}}_{0, c}\right)
$$

given by mapping $\bar{M}(\sigma)$ to its central character, is surjective.

Corollary $2.7\left(\left[12\right.\right.$, Corollary 5.10]). The morphism $\zeta_{0, c}^{*}: \operatorname{Irr}(W) \rightarrow \Upsilon^{-1}(0)$ from (2.5.6) is the determinant dual of the morphism $\Theta$ from (2.5.8), i.e.,

$$
\Theta(\sigma)=\zeta_{0, c}^{*}(\sigma \otimes \operatorname{det}), \text { for all } \sigma \in \operatorname{Irr}(W) \text {. }
$$

Remark 2.8. The partition of $\operatorname{Irr}(W)$ according to the fibers of the map $\Theta$ is called the Calogero-Moser (CM) partition. By Corollary 2.7, we know that this partition is the same as the Dirac partition, i.e., the one given by $\zeta_{0, c}^{*}$. This allows us to use Theorem 2.5 in order to study the CM partition.

\section{Classification of One-W-type Modules}

In order to apply Theorem 2.5, we construct modules for $\mathbf{H}_{0, c}$ for which the Dirac cohomology is easy to compute.

\subsection{Definition}

Let $(\pi, X)$ be a simple $\mathbf{H}_{0, c^{-}}$module. We consider simple modules $X$ such that the restriction of $X$ to $W$ is an irreducible $W$-representation. In the 
setting of graded affine Hecke algebras, this type of modules were introduced and classified in [3], and studied further in [13].

Given an irreducible $W$-representation $\left(\sigma, U_{\sigma}\right)$, the question is how to define the action of $\mathfrak{h}$ and $\mathfrak{h}^{*}$ in such a way that the commutation relations in $\mathbf{H}_{0, c}$ are preserved. Suppose $(\pi, X)$ is a one- $W$-type $\mathbf{H}_{0, c}$-module extending $\sigma$. As in [13, Appendix], consider the space

$$
\mathfrak{h} \cdot U_{\sigma}=\left\{\pi(y) u: y \in \mathfrak{h}, u \in U_{\sigma}\right\}
$$

Since $w \cdot y \cdot w^{-1}=w(y)$, the space $\mathfrak{h} \cdot U_{\sigma}$ is $W$-invariant. Moreover, it is a constituent of the $W$-representation refl $\otimes \sigma$, where refl is the reflection representation of $W$ on $\mathfrak{h}$. Since the restriction of $X$ to $W$ is irreducible, either $\mathfrak{h} \cdot U_{\sigma}=0$ or else $\mathfrak{h} \cdot U_{\sigma} \cong U_{\sigma}$ as $W$-representations. The latter case implies that

$$
\operatorname{Hom}_{W}[\sigma, \sigma \otimes \mathrm{refl}] \neq 0 .
$$

A similar analysis applies to $\mathfrak{h}^{*} \cdot U_{\sigma}$.

Remark 3.1. When $W$ is a finite Weyl group, [13, Appendix] determines all $W$-types $\sigma$ that satisfy condition (3.1.1). When the long Weyl group element $w_{0}$ is central in $W$, an easy argument implies that no such $\sigma$ exists. When $w_{0}$ is not central, i.e., types $A_{n-1}, D_{2 n+1}$, or $E_{6}$, there exist representations $\sigma$ satisfying (3.1.1). However, in the setting of the rational Cherednik algebra (unlike that of the graded affine Hecke algebra) we already know that for example in type $A$, no one- $W$-type modules can exist [14].

From now on, we assume that $W$ is a finite Weyl group. In light of Remark 3.1 and the discussion preceding it, we make the following definition.

Definition 3.2. A simple $\mathbf{H}_{0, c^{-}}$module $(\pi, X)$ is called a one- $W$-type module if

(1) the restriction of $X$ to $W$ is irreducible, and

(2) $\pi(y)=0=\pi(x)$ for all $y \in \mathfrak{h}, x \in \mathfrak{h}^{*}$.

Condition (2) is automatic when $w_{0}$ is central in $W$. 


\subsection{Criterion}

We determine which irreducible $W$-representations give rise to one- $W$ type modules in the sense of Definition 3.2. Let $R \subset \mathfrak{h}^{*}$ denote the set of roots, $R^{+}$, the set of positive roots, $\Pi$ the set of simple roots. Let $R^{\vee}$, $R^{+, \vee}$, and $\Pi^{\vee}$ denote the corresponding coroots in $\mathfrak{h}$. If $\alpha \in R$ is a root, let $\alpha^{\vee}$ be the corresponding coroot normalized such that $\left\langle\alpha, \alpha^{\vee}\right\rangle=2$. The (renormalized) basic commutation relation in $\mathbf{H}_{0, c}$ becomes

$$
[y, x]=-\sum_{\alpha>0} c_{\alpha}\langle y, \alpha\rangle\left\langle\alpha^{\vee}, x\right\rangle s_{\alpha}, \quad y \in \mathfrak{h}, x \in \mathfrak{h}^{*}
$$

Lemma 3.3. An irreducible $W$-representation $\sigma$ extends to a one-W-type $\mathbf{H}_{0, c}$-module if and only if

$$
\sum_{\alpha>0} c_{\alpha}\langle y, \alpha\rangle\left\langle\alpha^{\vee}, x\right\rangle \sigma\left(s_{\alpha}\right)=0,
$$

for all $y \in \mathfrak{h}$ and $x \in \mathfrak{h}^{*}$.

Proof. This is straightforward by Definition 3.2 and formula (3.2.1).

In order to carry out the computations effectively, we adapt a reduction criterion from [3, Proposition 2.4].

Proposition 3.4. Suppose $y \in \mathfrak{h}$ and $x_{1}, x_{2} \in \mathfrak{h}^{*}$ are such that

(a) $W \cdot y$ spans $\mathfrak{h}$ (modulo the $W$-invariants), and

(b) $\left\{x_{1}, Z_{W}(y) \cdot x_{2}\right\} \operatorname{span} \mathfrak{h}^{*}$.

The $W$-type $\sigma$ extends to a one-W-type module $(\pi, X)$ if and only if equation (3.2.2) holds for the pairs $\left(y, x_{1}\right)$ and $\left(y, x_{2}\right)$.

Proof. Without loss of generality, assume $R$ spans $\mathfrak{h}^{*}$. Suppose $\pi\left(\left[y, x_{1}\right]\right)=$ 0 and $\pi\left(\left[y, x_{2}\right]\right)=0$. Let $y^{\prime} \in \mathfrak{h}$ and $x^{\prime} \in \mathfrak{h}^{*}$ be arbitrary and we want to show that $\pi\left(\left[y^{\prime}, x^{\prime}\right]\right)=0$. For every $w \in W$, we have

$$
\left[w\left(y^{\prime}\right), w\left(x^{\prime}\right)\right]=w \cdot\left[y^{\prime}, x^{\prime}\right] \cdot w^{-1}
$$

Since $\pi\left(\left[y, x_{2}\right]\right)=0$ it follows from this that $\pi\left(\left[y, w\left(x_{2}\right)\right]\right)=0$ for all $w \in$ $Z_{W}(y)$. From (b), it follows that $\pi\left(\left[y, x^{\prime}\right]\right)=0$ for all $x^{\prime} \in \mathfrak{h}^{*}$. But then also 
$\pi\left(\left[w(y), x^{\prime}\right]\right)=0$ for all $w \in W$ and $x^{\prime} \in \mathfrak{h}^{*}$, and the conclusion follows from (a).

\subsection{Classification}

To describe the classification result, let us recall first the parameterization of irreducible $W$-representations. If $W=S_{n}$ is of type $A_{n-1}$, the $W$-types are parameterized by partitions $\lambda$ of $n$; we write $(\lambda)$ for the corresponding representation. In this notation, $(n)$ denotes the trivial representation and $\left(1^{n}\right)$ the sign representation. We also denote by $\lambda^{t}$ the transpose partition to $\lambda$.

If $W$ is of type $B_{n}$ (equivalently $C_{n}$ ), then $W_{n}=S_{n} \ltimes(\mathbb{Z} / 2 \mathbb{Z})^{n}$ and the irreducible $W$-representations are parameterized by bipartitions $(\lambda, \mu)$ of $n$ via Mackey induction. We denote by $(\lambda) \times(\mu)$ the $W$-type

$$
(\lambda) \times(\mu)=\operatorname{Ind}_{S_{n-k} \times S_{k} \times(\mathbb{Z} / 2 \mathbb{Z})^{n}}^{S_{n}}\left((\lambda) \otimes(\mu) \otimes \operatorname{triv}^{\otimes(n-k)} \otimes \operatorname{sgn}^{\otimes k}\right) .
$$

If $R$ is of type $D_{n}, W\left(D_{n}\right)$ is an index 2 subgroup of $W\left(B_{n}\right)$ and the irreducible $W\left(D_{n}\right)$-representations are obtained by restriction from $W\left(B_{n}\right)$. If $\lambda \neq \mu$, then $(\lambda) \times(\mu)$ and $(\mu) \times(\lambda)$ restrict to the same $W\left(D_{n}\right)$-representation, which we denote again by $(\lambda) \times(\mu)$. When $\lambda=\mu$, the restriction of the $W\left(B_{n}\right)$-type $(\lambda) \times(\lambda)$ to $W\left(D_{n}\right)$ splits into a sum of two equidimensional, non isomorphic representations, denoted $(\lambda) \times(\lambda)_{I}$ and $(\lambda) \times(\lambda)_{I I}$.

When $W$ is of exceptional type, we will use the notation of Kondo for $F_{4}$ and Frame for $E_{6}, E_{7}, E_{8}$, see [20], and Carter's notation for $G_{2}$ [11].

If the parameter function $c$ for the rational Cherednik algebra $\mathbf{H}_{0, c}$ is identically zero, then every $W$-type extends trivially to an $\mathbf{H}_{0, c}$-module. We assume this is not the case from now on. Thus, without loss of generality, we may assume $c$ is identically 1 if the root system is simple and simplylaced. If the simple root system $R$ has two root lengths, we denote by $c_{s}$ the parameter on the short roots and by $c_{\ell}$ the parameter on the long roots. When the algebra if of type $B / C$, this convention refers to the type $B_{n}$ root system.

Theorem 3.5. Let $R$ be a simple root system. The irreducible $W$ representations that extend to a one-W-type $\mathbf{H}_{0, c}$-module in the sense of Definition 3.2 are: 
(1) $\mathbf{A}_{\mathbf{n}-\mathbf{1}}$ or $\mathbf{E}_{\mathbf{7}}$ : none;

(2) $\mathbf{B}_{\mathbf{n}}$ : the $W$-representations of the form $(\lambda) \times(0)$ and $(0) \times\left(\lambda^{t}\right)$, where $\lambda$ is a rectangular partition of $n$ of the form $\lambda=(\underbrace{d, d, \ldots, d}_{k})$ with

$$
k-d=c_{s} / c_{\ell}
$$

(3) $\mathbf{D}_{\mathbf{n}}$ : the $W$-representations of the form $(\lambda) \times(0)$ where $\lambda$ is a rectangular partition of $n$ of the form $\lambda=(\underbrace{d, d, \ldots, d}_{d})$ (so $\left.n=d^{2}\right)$;

(4) $\mathbf{E}_{\mathbf{6}}$ : the representation $10_{s}$;

(5) $\mathbf{E}_{\mathbf{8}}$ : the representations $168_{y}$ and $420_{y}$;

(6) $\mathbf{G}_{\mathbf{2}}$ :

(a) $\phi_{1,3}^{\prime}, \phi_{1,3}^{\prime \prime}$, and $\phi_{2,2}$ when $c_{s} / c_{\ell}=1$;

(b) $\phi_{1,0}, \phi_{1,6}$, and $\phi_{2,1}$ when $c_{s} / c_{\ell}=-1$;

(7) $\mathbf{F}_{4}$ :

(a) $4_{1}$ for all values of the parameters $c_{s}, c_{\ell}$;

(b) $1_{2}, 1_{3}, 6_{1}, 4_{3}, 4_{4}$ when $c_{s} / c_{\ell}=1$;

(c) $1_{1}, 1_{4}, 6_{2}, 4_{2}, 4_{5}$ when $c_{s} / c_{\ell}=-1$;

(d) $2_{1}, 2_{2}$ when $c_{\ell}=0$;

(e) $2_{3}, 2_{4}$ when $c_{s}=0$;

In the rest of the section, we present the proof of Theorem 3.5 .

\subsection{Classical types}

We write the roots of root system of classical types in the standard coordinates. For simplicity, we work with the algebra of type $g l(n)$ in type $A$. Let $\left\{\epsilon_{1}, \ldots, \epsilon_{n}\right\}$ be the standard coordinates for $\mathfrak{h}^{*}$ and $\left\{\epsilon_{1}^{\vee}, \ldots, \epsilon_{n}^{\vee}\right\}$ the dual coordinates for $\mathfrak{h}$. The positive roots are denoted as follows:

(1) $\mathbf{A}_{\mathbf{n}-\mathbf{1}}: \epsilon_{i}-\epsilon_{j}, 1 \leq i<j \leq n$;

(2) $\mathbf{B}_{\mathbf{n}}: \epsilon_{i} \pm \epsilon_{j}, 1 \leq i<j \leq n$, and $\epsilon_{i}, 1 \leq i \leq n$;

(3) $\mathbf{D}_{\mathbf{n}}: \epsilon_{i} \pm \epsilon_{j}, 1 \leq i<j \leq n$. 
In the notation of Proposition 3.4, we choose in all cases

$$
y=\epsilon_{1}^{\vee}, \quad x_{1}=\epsilon_{1}, \quad x_{2}=\epsilon_{2}
$$

Suppose $\sigma$ is a $W$-type that can be extended to a one- $W$-type module. The two conditions from Proposition 3.4 imply in type $A_{n-1}$ :

$$
\sum_{i=2}^{n} \sigma\left(s_{\epsilon_{1}-\epsilon_{i}}\right)=0 \text { and } \sigma\left(s_{\epsilon_{1}-\epsilon_{2}}\right)=0 .
$$

But clearly the second condition is impossible, so there are no such $S_{n^{-}}$ representations.

Suppose that we are in type $B_{n}$ now. The two conditions become

$$
\begin{aligned}
& c_{\ell} \sum_{i=1}^{n}\left(\sigma\left(s_{\epsilon_{1}-\epsilon_{i}}\right)+\sigma\left(s_{\epsilon_{1}+\epsilon_{i}}\right)\right)+2 c_{s} \sigma\left(s_{\epsilon_{1}}\right)=0 ; \\
& c_{\ell}\left(-\sigma\left(s_{\epsilon_{1}-\epsilon_{2}}\right)+\sigma\left(s_{\epsilon_{1}+\epsilon_{2}}\right)\right)=0 .
\end{aligned}
$$

If $c_{\ell}=0$, the two conditions imply that $c_{s} \sigma\left(s_{\epsilon_{1}}\right)=0$, which is impossible unless $c_{s}=0$. So suppose $c_{\ell} \neq 0$, and set $c=c_{s} / c_{\ell}$. The second condition implies that $\sigma\left(s_{\epsilon_{1}-\epsilon_{2}}\right)=\sigma\left(s_{\epsilon_{1}+\epsilon_{2}}\right)$ and by conjugation that

$$
\sigma\left(s_{\epsilon_{i}-\epsilon_{j}}\right)=\sigma\left(s_{\epsilon_{i}+\epsilon_{j}}\right)=\sigma\left(s_{\epsilon_{j}}\right) \sigma\left(s_{\epsilon_{i}-\epsilon_{j}}\right) \sigma\left(s_{\epsilon_{j}}\right),
$$

for all $i<j$. But this means that every $\sigma\left(s_{\epsilon_{j}}\right)$ preserves with the restriction of $\sigma$ to $S_{n}$, and since $\sigma$ is irreducible, it must be of the form $(\lambda) \times(0)$ or $(0) \times\left(\lambda^{t}\right)$.

The two cases are dual to each other via tensoring with the sign representation, so it is sufficient to treat the first one. If $\sigma=(\lambda) \times(0)$, then $\sigma\left(s_{\epsilon_{i}}\right)=\operatorname{Id}$ for all $i$ and $\sigma\left(s_{\epsilon_{i}-\epsilon_{j}}\right)=(\lambda)\left(s_{\epsilon_{i}-\epsilon_{j}}\right)$. The condition is then one in $S_{n}$ :

$$
\sum_{i=2}^{n}(\lambda)\left(s_{\epsilon_{1}-\epsilon_{i}}\right)+c \cdot \operatorname{Id}=0 .
$$

A similar condition appears in [3] in the setting of the graded Hecke algebra of type $A$, see the proof of Theorem 3.15, particularly (3.18) in [3]. One can 
compute it using the realization of $S_{n}$-representations in harmonic polynomials and the result is that (3.4.4) holds if and only if $\lambda$ is a rectangular partition $\lambda=(\underbrace{d, \ldots, d}_{k})$ satisfying

$$
c=k-d
$$

In type $D_{n}$, the two necessary and sufficient conditions are:

$$
\begin{aligned}
& \sum_{i=1}^{n}\left(\sigma\left(s_{\epsilon_{1}-\epsilon_{i}}\right)+\sigma\left(s_{\epsilon_{1}+\epsilon_{i}}\right)\right)=0 \\
& -\sigma\left(s_{\epsilon_{1}-\epsilon_{2}}\right)+\sigma\left(s_{\epsilon_{1}+\epsilon_{2}}\right)=0 .
\end{aligned}
$$

The discussion in this case is identical with the case $B_{n}$ with $c_{\ell}=1$ and $c_{s}=0$.

\subsection{Exceptional types}

For the exceptional simple root systems, we would like to use characters of representations (rather than the representations themselves), so we need to adapt Proposition 3.4. The idea is the same as in [3, Proposition 4.1]. If $\sigma$ is a $W$-representation, let $\chi_{\sigma}$ denote its character.

Proposition 3.6. Let $\sigma$ be an irreducible $W$-representation and $y, x_{1}, x_{2}$ as in Proposition 3.4. Suppose that the parameters $c$ are real. Then $\sigma$ extends to a one-W-type $\mathbf{H}_{0, c}$-module if and only if

$$
\chi_{\sigma}\left(\left[y, x_{i}\right]^{2}\right)=0, \quad i=1,2 .
$$

Proof. We need to show that $\sigma([y, x])=0$ if and only if $\chi_{\sigma}\left([y, x]^{2}\right)=0$. We can realize $\sigma\left(s_{\alpha}\right)$ as a symmetric real-valued (in fact, rational) matrix for every $\alpha>0$. This means that $\sigma([y, x])$ is also a real-valued symmetric matrix and therefore, it is diagonalizable and has real eigenvalues $\nu_{i}, i=1, \operatorname{dim} \sigma$. But then $\chi_{\sigma}\left([y, x]^{2}\right)=\sum_{i} \nu_{i}^{2}=0$ if and only if $\nu_{i}=0$ for all $i$.

The explicit calculations are as follows. For each exceptional simple root system, we choose $y, x_{1}, x_{2}$ and compute $\left[y, x_{i}\right], i=1,2$, as combination of $s_{\alpha}, \alpha>0$. Next we compute the squares $\left[y, x_{i}\right]^{2}$ which are linear combination of terms $s_{\alpha} s_{\beta}$. For the character computation in Proposition 3.6, the only important feature is which rank 2 root subsystem every such pair $\alpha, \beta$ forms. 
Thus we need to count all the occurrences of a given rank 2 subsystem in this combination. We used the computer algebra systems "Mathematica" to carry out these straightforward, but tedious root calculations and GAP version 3.4 [15] with the package "chevie" [17] to compute the characters.

It turns out that in all cases, except in $F_{4}$, if there exists a $W$-type $\sigma$ such $\chi_{\sigma}\left(\left[y, x_{i}\right]^{2}\right)=0, i=1,2$, then necessarily the parameters $c$ are real (and are very restrictive). Since the condition in Proposition 3.6 is clearly necessary even when the parameters are complex, it follows that in those cases, we don't need to check the nonreal parameters separately. In $F_{4}$, there exists two cases when $\chi_{\sigma}\left(\left[y, x_{i}\right]^{2}\right)=0$, and there we do a different calculation.

We summarize the calculations next. We will denote by $w_{A_{2}}, w_{\widetilde{A}_{2}}, w_{2 A_{1}}$, $w_{A_{1}+\widetilde{A}_{1}}, w_{B_{2}}, w_{G_{2}}$ representatives of the conjugacy classes of the corresponding rank 2 root subsystems.

3.5.1. $G_{2}$

Let $R$ be of type $G_{2}$. We choose the coordinates for the simple roots:

$$
\alpha_{s}=(2 / 3,-1 / 3,-1 / 3), \quad \alpha_{\ell}=(-1,1,0),
$$

and

$$
y=(1,1,-2), \quad x_{1}=(1 / 3,1 / 3,-2 / 3), \quad x_{2}=(0,1,-1) .
$$

Then $\left[y, x_{1}\right]$ and $\left[y, x_{2}\right]$ have 5 and 4 terms, respectively. Calculating the quantities in Proposition 3.6. we find that $\sigma$ extends to a one- $W$-type module if and only if $\chi_{\sigma}$ vanishes on

$$
\begin{aligned}
& 3\left(c_{\ell}^{2}+c_{s}^{2}\right) 1+\left(2 c_{\ell} c_{s}\right) w_{A_{1}+\widetilde{A}_{1}}+3\left(c_{\ell}^{2}+c_{s}^{2}\right) w_{A_{2}}+\left(10 c_{\ell} c_{s}\right) w_{G_{2}}, \text { and } \\
& 5\left(c_{\ell}^{2}+c_{s}^{2}\right) 1+\left(2 c_{\ell} c_{s}\right) w_{A_{1}+\widetilde{A}_{1}}+4\left(c_{\ell}^{2}+c_{s}^{2}\right) w_{A_{2}}+\left(16 c_{\ell} c_{s}\right) w_{G_{2}} .
\end{aligned}
$$

Using the character table for $G_{2}$ (in GAP), we verify Theorem 3.5 for $G_{2}$.

3.5.2. $F_{4}$

Let $R$ be of type $F_{4}$. We choose the following coordinates for the simple roots:

$$
\alpha_{1}=\epsilon_{1}-\epsilon_{2}-\epsilon_{3}-\epsilon_{4}, \alpha_{2}=2 \epsilon_{4}, \alpha_{3}=\epsilon_{3}-\epsilon_{4}, \alpha_{4}=\epsilon_{2}-\epsilon_{3}
$$

and 


$$
y=\epsilon_{1}^{\vee}, \quad x_{1}=\epsilon_{1}, \quad x_{2}=\epsilon_{1}+\epsilon_{2}+\epsilon_{3}+\epsilon_{4}
$$

Then $\left[y, x_{1}\right]$ has 15 terms and $\left[y, x_{2}\right]$ has 9 terms. Calculating the squares, we find that $\sigma$ extends to a one- $W$-type module if and only if $\chi_{\sigma}$ vanishes on

$$
\begin{aligned}
& \left(c_{\ell}^{2}+c_{s}^{2}\right) 1+\left(c_{\ell}^{2}+c_{s}^{2}\right) w_{2 A_{1}}+\left(4 c_{\ell} c_{s}\right) w_{A_{1}+\widetilde{A}_{1}}+\left(4 c_{\ell}^{2}\right) w_{A_{2}}+\left(4 c_{s}^{2}\right) w_{\widetilde{A}_{2}}+\left(8 c_{\ell} c_{s}\right) w_{B_{2}} ; \\
& \left(c_{\ell}^{2}+c_{s}^{2}\right) 1+\left(2 c_{\ell}^{2}\right) w_{A_{2}}+\left(2 c_{s}^{2}\right) w_{\widetilde{A}_{2}}+\left(6 c_{\ell} c_{s}\right) w_{B_{2}} .
\end{aligned}
$$

Using GAP, we extract the character table of $W\left(F_{4}\right)$ for these conjugacy classes, and verify Theorem 3.5 in this case. It turns out that in this case if $c_{s} / c_{\ell}= \pm \sqrt{-1}$, then also $12_{1}$ and $16_{1}$ have the property that $\chi\left(\left[y, x_{i}\right]^{2}\right)=0$. Since the characters of both $12_{1}$ and $16_{1}$ vanish on the reflections, it follows that in fact $\chi\left(\left[y, x_{i}\right]\right)=0$ as well. In order to settle this case, we compute $\left[y, x_{1}\right]$ explicitly using matrix realizations $1 \|$ of $12_{1}$ and $16_{1}$ and we find that (3.2.2) does not hold for these two representations for any values of $c_{s}, c_{\ell}$.

\subsection{3. $E_{6}$}

Let $R$ be of type $E_{6}$. We assume the parameters equal to 1 . The simple roots are

$$
\begin{aligned}
& \alpha_{1}=\frac{1}{2}(1,-1,-1,-1,-1,-1,-1,1), \alpha_{2}=\epsilon_{1}+\epsilon_{2}, \alpha_{3}=-\epsilon_{1}+\epsilon_{2}, \\
& \alpha_{4}=-\epsilon_{2}+\epsilon_{3}, \alpha_{5}=-\epsilon_{3}+\epsilon_{4}, \alpha_{6}=-\epsilon_{4}+\epsilon_{5} .
\end{aligned}
$$

Let $\omega_{i}, \omega_{i}^{\vee}$ be the fundamental weights, respectively coweights. We choose

$$
y=\omega_{2}^{\vee}, \quad x_{1}=\omega_{2}, \quad x_{2}=s_{\alpha_{2}}\left(x_{1}\right) .
$$

Then $\left[y, x_{1}\right]$ has 21 terms and $\left[y, x_{2}\right]$ has 12 terms. Calculating the squares, we find that $\sigma$ extends to a one- $W$-type module if and only if $\chi_{\sigma}$ vanishes on

$$
1+5 w_{2 A_{1}}+10 w_{A_{2}} \text { and } 1+w_{2 A_{1}}+6 w_{A_{2}} .
$$

Using GAP, we extract the character table of $W\left(E_{6}\right)$ for the conjugacy classes $1,2 A_{1}$ and $A_{2}$, and find that the only $W$-type whose character vanishes is $10_{s}$.

\footnotetext{
${ }^{1}$ We used J. Adams' matrix models of Weyl group representations that can be found at http://www.liegroups.org/weyl/integral/
} 


\subsection{4. $E_{7}$}

Let $R$ be of type $E_{7}$. We assume the parameters equal to 1 . The simple roots are

$\alpha_{1}=\frac{1}{2}(1,-1,-1,-1,-1,-1,-1,1), \alpha_{2}=\epsilon_{1}+\epsilon_{2}, \alpha_{3}=-\epsilon_{1}+\epsilon_{2}$,

$\alpha_{4}=-\epsilon_{2}+\epsilon_{3}, \alpha_{5}=-\epsilon_{3}+\epsilon_{4}, \alpha_{6}=-\epsilon_{4}+\epsilon_{5}, \alpha_{7}=-\epsilon_{5}+\epsilon_{6}$.

We choose

$$
y=\omega_{1}^{\vee}, \quad x_{1}=\omega_{1}, \quad x_{2}=s_{\alpha_{1}}\left(x_{1}\right) .
$$

Then $\left[y, x_{1}\right]$ has 33 terms and $\left[y, x_{2}\right]$ has 18 terms. Calculating the squares, we find that $\sigma$ extends to a one- $W$-type module if and only if $\chi_{\sigma}$ vanishes on

$$
1+10 w_{2 A_{1}}+16 w_{A_{2}} \text { and } 2+5 w_{2 A_{1}}+20 w_{A_{2}} .
$$

Using GAP, we extract the character table of $W\left(E_{7}\right)$ for the conjugacy classes $1,2 A_{1}$ and $A_{2}$, and find that no $W$-characters vanish on these elements.

\subsection{5. $E_{8}$}

Let $R$ be of type $E_{8}$. We assume the parameters equal to 1 . The simple roots are

$$
\begin{aligned}
& \alpha_{1}=\frac{1}{2}(1,-1,-1,-1,-1,-1,-1,1), \alpha_{2}=\epsilon_{1}+\epsilon_{2}, \alpha_{3}=-\epsilon_{1}+\epsilon_{2}, \\
& \alpha_{4}=-\epsilon_{2}+\epsilon_{3}, \alpha_{5}=-\epsilon_{3}+\epsilon_{4}, \alpha_{6}=-\epsilon_{4}+\epsilon_{5}, \alpha_{7}=-\epsilon_{5}+\epsilon_{6} \alpha_{8}=-\epsilon_{6}+\epsilon_{7} .
\end{aligned}
$$

We choose

$$
y=\omega_{8}^{\vee}, \quad x_{1}=\omega_{8}, \quad x_{2}=s_{\alpha_{8}}\left(x_{1}\right) .
$$

Then $\left[y, x_{1}\right]$ has 57 terms and $\left[y, x_{2}\right]$ has 30 terms. Calculating the squares, we find that $\sigma$ extends to a one- $W$-type module if and only if $\chi_{\sigma}$ vanishes on

$$
1+21 w_{2 A_{1}}+28 w_{A_{2}} \text { and } 1+6 w_{2 A_{1}}+18 w_{A_{2}} .
$$

Using GAP, we extract the character table of $W\left(E_{8}\right)$ for the conjugacy classes $1,2 A_{1}$ and $A_{2}$, and find that the only $W$-characters that vanish on these elements are $168_{y}$ and $420_{y}$. 


\section{Dirac Cohomology and Cuspidal Two-sided Cells}

We retain the notation from sections 2 and 3 .

\subsection{One- $W$-type modules}

The Dirac cohomology of a one- $W$-type module can be computed easily. Proposition 4.1. Let $(\pi, X)$ be a one-W-type $\mathbf{H}_{0, c}$-module that extends the irreducible $W$-representation $\sigma$. Then $H_{D}(X)=\sigma \otimes \wedge \mathfrak{h}$.

Proof. Since $X$ is a one- $W$-type module, every $x \in \mathfrak{h}^{*}$ and $y \in \mathfrak{h}$ act by 0 on $X$. Since $\mathcal{D}=\sum_{i} x_{i} \otimes y_{i}+\sum_{i} y_{i} \otimes x_{i}$, it follows that $\mathcal{D}$ also acts by 0 on $X \otimes S$, and so ker $D_{X}=X \otimes S$ while im $D_{X}=0$. Thus $H_{D}(X)=X \otimes S$ as $W$-representations and the claim follows.

Recall the partition of IrrW into Calogero-Moser cells, i.e., the fibers of the map $\Theta$ from (2.5.8). If $\sigma, \sigma^{\prime} \in \operatorname{Irr} W$, denote by

$$
\sigma \sim_{C M} \sigma^{\prime} \text { if } \Theta(\sigma)=\Theta\left(\sigma^{\prime}\right) \in \Upsilon^{-1}(0)
$$

Denote also by $[\sigma]_{C M}$ the Calogero-Moser cell containing $\sigma$.

Corollary 4.2. Let $\sigma$ be an irreducible $W$-representation that can be extended to a one-W-type module. If $\sigma^{\prime} \in \operatorname{Irr} W$ is such that $\operatorname{Hom}_{W}\left[\sigma^{\prime}, \sigma \otimes\right.$ $\bigwedge \mathfrak{h}] \neq 0$, then $\sigma \sim_{C M} \sigma^{\prime}$.

Proof. If $\operatorname{Hom}_{W}\left[\sigma^{\prime}, \sigma \otimes \wedge \mathfrak{h}\right] \neq 0$, then $\operatorname{Hom}_{W}\left[\sigma^{\prime} \otimes \operatorname{sgn}, \sigma \otimes \wedge \mathfrak{h}\right] \neq 0$ because $\bigwedge \mathfrak{h} \otimes$ sgn $=\bigwedge \mathfrak{h}$. It follows from Proposition 4.1 that $\operatorname{Hom}_{W}\left[\sigma^{\prime} \otimes\right.$ sgn, $\left.H_{D}(X)\right] \neq 0$. (In particular, this applies for $\sigma$ too.) By Theorem 2.5 , $\zeta_{0, c}^{*}(\sigma \otimes \mathrm{sgn})=\zeta_{0, c}^{*}\left(\sigma^{\prime} \otimes \mathrm{sgn}\right)$. Corollary 2.7 then says that $\Theta(\sigma)=\Theta\left(\sigma^{\prime}\right)$.

\subsection{Cuspidal two-sided cells}

We restrict to the case when the parameters $c$ are equal to 1 and recall the necessary definitions from [20].

If $\sigma \in \operatorname{Irr}(W)$, let $a(\sigma) \geq 0$ denote the (integer) exponent of the smallest power of $q$ that appears in expansion of the formal degree of the Hecke 
algebra representation corresponding to $\sigma$. (See [20, (4.1.1) and §3.1].) Let $b(\sigma)$ denote the smallest nonnegative integer such that $\sigma$ occurs in the $i$-th symmetric power of the reflection representation. A result of Lusztig says that $a(\sigma) \leq b(\sigma)$ always, and a representation $\sigma$ is called special if equality holds.

Before discussing the partition of IrrW into double cells, we record the following remarkable result that will be needed later in connection with the formula for $\mathcal{D}^{2}$ from Proposition 2.3. This fact was noticed empirically by Beynon-Lusztig [8] and proved uniformly by Opdam [23].

Theorem 4.3. ([8, Propositions A and B],[23, Corollary 9]). Let $W$ be a finite reflection group and $\sigma$ an irreducible $W$-representation. The element $\Omega_{W, 1}=\sum_{\alpha>0} s_{\alpha}$ acts in $\sigma$ by

$$
N(\sigma)=a(\sigma \otimes \mathrm{sgn})-a(\sigma)
$$

If $W^{\prime}$ is a parabolic subgroup of $W$ and $\mu \in \operatorname{Irr} W^{\prime}$, define the truncated induction $([20,(4.1 .7)])$ :

$$
J_{W^{\prime}}^{W}(\mu)=\sum_{\sigma}\langle\mu, \sigma\rangle_{W^{\prime}} \sigma
$$

where the sum ranges over all $\sigma \in \operatorname{Irr} W$ such that $a(\sigma)=a(\mu)$. (If $\langle\mu, \sigma\rangle_{W^{\prime}} \neq$ 0 , then necessarily $a(\sigma) \geq a(\mu)$.)

Following [20, $\S 4.2]$, define inductively a partition of $\operatorname{lr} W$ into families (or two-sided cells) of representations. If $W=\{1\}$, then there is only one family consisting of the trivial representation. Assume that $W \neq\{1\}$ and that we have defined families for all parabolic subgroups $W^{\prime} \neq W$. Then two $W$-representations $\sigma$ and $\sigma^{\prime}$ are in the same family for $W$ if there exists a sequence of $W$-representations

$$
\sigma=\sigma_{0}, \sigma_{1}, \ldots, \sigma_{m}=\sigma^{\prime}
$$

such that for every $i, 0 \leq i \leq m-1$ there exists a parabolic subgroup $W_{i} \neq W$ and $W_{i}$-representations $\mu_{i}^{\prime}, \mu_{i}^{\prime \prime}$ in the same family for $W_{i}$ such that either

$$
\left\langle\mu_{i}^{\prime}, \sigma_{i-1}\right\rangle_{W_{i}} \neq 0, a\left(\mu_{i}^{\prime}\right)=a\left(\sigma_{i-1}\right) \text { and }\left\langle\mu_{i}^{\prime \prime}, \sigma_{i}\right\rangle_{W_{i}} \neq 0, a\left(\mu_{i}^{\prime \prime}\right)=a\left(\sigma_{i}\right) \text {, }
$$


or

$$
\begin{aligned}
& \left\langle\mu_{i}^{\prime}, \sigma_{i-1} \otimes \operatorname{sgn}\right\rangle_{W_{i}} \neq 0, a\left(\mu_{i}^{\prime}\right)=a\left(\sigma_{i-1} \otimes \operatorname{sgn}\right) \text { and } \\
& \left\langle\mu_{i}^{\prime \prime}, \sigma_{i} \otimes \operatorname{sgn}\right\rangle_{W_{i}} \neq 0, a\left(\mu_{i}^{\prime \prime}\right)=a\left(\sigma_{i} \otimes \operatorname{sgn}\right) .
\end{aligned}
$$

From the definition one sees that if $\mathcal{F}$ is a family in $\operatorname{Irr} W$ then so is $\mathcal{F} \otimes$ sgn. Moreover, from Theorem 4.3, we see that the scalars $N(\sigma)$ are constant as $\sigma$ varies in a fixed family.

The description of the families for each irreducible Weyl group is made explicit in [20, Chapter 4]. In the case of classical groups other than type $A$, the characterization of families is in terms of certain symbols, see [20, $\S 4.5-\S 4.7]$

We also recall that each family contains a unique special representation.

Definition $4.4([20, \S 8.1])$. A family $\mathcal{F} \subset \operatorname{Irr} W$ is called non-cuspidal if there exists a proper parabolic subgroup $W^{\prime}$ of $W$ and a family $\mathcal{F}^{\prime} \subset \operatorname{Irr} W^{\prime}$ such that either

(a) $J_{W^{\prime}}^{W}$ establishes a bijection between $\mathcal{F}^{\prime}$ and $\mathcal{F}$ or

(b) $J_{W^{\prime}}^{W}$ establishes a bijection between $\mathcal{F}^{\prime}$ and $\mathcal{F} \otimes$ sgn.

A family $\mathcal{F} \subset \operatorname{Ir} W$ is called cuspidal if it is not non-cuspidal.

Theorem $4.5([20, \S 8])$. The classification of cuspidal families is as follows.

(1) Type $\mathbf{A}_{\mathbf{n}-\mathbf{1}}$ : no cuspidal families.

(2) Type $\mathbf{B}_{\mathbf{n}}$ : only when $n=d^{2}+d$ for some $d \geq 1$ and then there is a unique cuspidal family for which the special representation is in bipartition notation $(1,2, \ldots, d) \times(1,2, \ldots, d)$ and the symbol is

$$
\left(\begin{array}{lllll}
0 & 2 & & \cdots & 2 d \\
& 1 & 3 & \cdots & 2 d-1
\end{array}\right) .
$$

(3) Type $\mathbf{D}_{\mathbf{n}}$ : only when $n=d^{2}$ for some $d \geq 2$ in which case there is a unique cuspidal family for which the special representation is in bipartition notation $(1,2, \ldots, d) \times(1,2, \ldots, d-1)$ and the symbol is $\left(\begin{array}{lllll}0 & 2 & 4 & \ldots & 2 d-2 \\ 1 & 3 & 5 & \ldots & 2 d-1\end{array}\right)$. 
(4) Type $\mathbf{G}_{\mathbf{2}}$ : the family $\left\{\phi_{2,1}, \phi_{2,2}, \phi_{1,3}^{\prime}, \phi_{1,3}^{\prime \prime}\right\}$;

(5) Type $\mathbf{F}_{\mathbf{4}}$ : the family $\left\{12_{1}, 9_{2}, 9_{3}, 1_{2}, 1_{3}, 4_{1}, 4_{3}, 4_{4}, 6_{1}, 6_{2}, 16_{1}\right\}$;

(6) Type $\mathbf{E}_{\mathbf{6}}$ : the family $\left\{80_{s}, 60_{s}, 90_{s}, 10_{s}, 20_{s}\right\}$;

(7) Type $\mathbf{E}_{\mathbf{7}}$ : the family $\left\{512_{a}^{\prime}, 512_{a}\right\}$;

(8) Type $\mathbf{E}_{\mathbf{8}}$ : the family $\left\{4480_{y}, 3150_{y}, 4200_{y}, 4536_{y}, 5670_{y}, 420_{y}, 1134_{y}\right.$, $\left.1400_{y}, 2688_{y}, 1680_{y}, 168_{y}, 70_{y}, 7168_{w}, 1344_{w}, 2016_{w}, 5600_{w}, 448_{w}\right\}$.

For the exceptional types, the first representation listed in the family is the special one.

Denote by $\mathcal{F}_{\text {cusp }}(W)$ the unique cuspidal family of $W$ if this exists. (Otherwise, $\mathcal{F}_{\text {cusp }}(W)=\emptyset$.)

\subsection{Induced representations}

As mentioned in the introduction, the reason one- $W$-type modules are intrinsically related to cuspidal Calogero-Moser points is Bellamy's result [4, Corollary 1] combined with the known fact that an irreducible Weyl group representation is not induced from a proper parabolic subgroup. I do not know a uniform proof of this fact. 2 Recall that the complex linear span $R(W)_{\text {Ind }}$ of all induced characters from proper parabolic subgroups can also be identified with the space of class functions on $W$ that are zero on the elliptic conjugacy classes. An element $w \in W$ is called elliptic if $w$ has no nonzero fixed points in the reflection $W$-representation.

If the longest Weyl group element $w_{0}$ is central in $W$, then $w_{0}$ is elliptic. In this case, for every irreducible $W$-representation $\sigma, \chi_{\sigma}\left(w_{0}\right)=$ $(-1)^{d} \operatorname{dim} \sigma \neq 0$, where $d$ is the lowest harmonic degree of $\sigma$. In particular, $\sigma \notin R(W)_{\text {Ind }}$, hence it is also not proper parabolically induced.

But when $w_{0}$ is not central, this argument fails. For example, in type $A_{n-1}$, the only elliptic conjugacy class is the $n$-cycles and it is well known

\footnotetext{
${ }^{2}$ For irreducible complex reflection groups (except maybe for $W\left(E_{6}\right)=G_{35}$ ), this is verified in 7 , Theorem 4.2], via a case-by-case argument too.
} 
that for a Young representation $\lambda$,

$$
\chi_{\lambda}((1,2, \ldots, n))= \begin{cases}(-1)^{m}, & \lambda=(n-m, 1, \ldots, 1) \\ 0, & \text { otherwise }\end{cases}
$$

Hence all irreducible $S_{n}$-representations, other than the wedges of the reflection representation, are in $R\left(S_{n}\right)_{\text {Ind }}$.

Similarly, in type $E_{6}$, there are 5 elliptic conjugacy classes and one can verify that there are two irreducible characters of $W\left(E_{6}\right)$ that vanish on all of them: $81_{p}$ and $81_{p}^{\prime}$.

Thus to complete the verification of the fact, we need certain ad-hoc arguments. For $A_{n-1}$, one may see easily from the Littlewood-Richardson rule that a proper parabolically induced representation is never irreducible. For $D_{n}$ (in fact, we only need $n$ odd), again the corresponding variant of the Littlewood-Richardson rule works. For $E_{6}$, we can check the fact by a direct computation with GAP/chevie or by consulting the induction/restriction tables of Alvis [1]. It is entertaining to remark that $\operatorname{Ind}_{D_{5}}^{E_{6}}((311) \times 0)=$ $81_{p}+81_{p}^{\prime}$

\subsection{Cuspidal Calogero-Moser points}

Assume the parameter $c$ of the rational Cherednik algebra is identically equal to 1 (equivalently, any constant). Denote by $\mathbf{H}_{0,1}$ this equal parameter algebra. If $\mathbf{H}_{0,1}$ has one- $W$-type modules, then in these modules every $x \in \mathfrak{h}^{*}$ and $y \in \mathfrak{h}$ act by zero. Let

$$
\mathbf{0} \in \Upsilon^{-1}(0) \subset X_{c}(W)
$$

be the corresponding point in the Calogero-Moser space. We now combine Corollary 4.2 with the classification of one- $W$-type modules from Theorem 3.5

Theorem 4.6. Let $\mathbf{H}_{0,1}$ be the equal parameter rational Cherednik algebra $($ at $t=0)$ for a simple finite Weyl group $W$.

(1) If $W$ is of type $B_{n}$ and $n=d^{2}+d$, then $\Theta^{-1}(\mathbf{0}) \supseteq \mathcal{F}_{\text {cusp }}\left(B_{n}\right)$.

(2) If $W$ is of type $D_{n}$ and $n=d^{2}$, then $\Theta^{-1}(\mathbf{0}) \supseteq \mathcal{F}_{\text {cusp }}\left(D_{n}\right)$. 
(3) If $W$ is of type $G_{2}, F_{4}$, or $E_{6}$, then $\Theta^{-1}(\mathbf{0})=\mathcal{F}_{\text {cusp }}(W)$.

(4) If $W$ is of type $E_{8}$, then $\mathcal{F}_{\text {cusp }}(W) \backslash\left\{4480_{y}\right\} \subseteq \Theta^{-1}(\mathbf{0}) \subseteq \mathcal{F}_{\text {cusp }}(W) \cup$ $\left\{2100_{y}\right\}$.

Proof. Let $\sigma$ be an irreducible $W$-representation that affords a one- $W$-type module. Then Corollary 4.2 implies that every irreducible constituent of $\sigma \otimes \wedge \mathfrak{h}$ is contained in $\Theta^{-1}(\mathbf{0})$. We discuss each case as in Theorem 3.5 . when the parameters are equal.

Type $\mathbf{B}_{\mathbf{n}}$. Let $n=d^{2}+d$ consider $\sigma=(\underbrace{d, \ldots, d}_{d+1}) \times(0)$. The other case is just $\sigma \otimes$ sgn so it gives the same result. We need to decompose $\sigma \otimes \wedge \mathfrak{h}$. The wedge representations in type $B_{n}$ are

$$
\bigwedge^{\ell} \mathfrak{h}=(n-\ell) \times(\ell)=\operatorname{Ind}_{S_{n-\ell} \times S_{\ell} \times(\mathbb{Z} / 2 \mathbb{Z})^{n}}^{W\left(B_{n}\right)}\left((n-\ell) \otimes(\ell) \otimes 1^{\otimes(n-\ell)} \otimes \epsilon^{\otimes \ell}\right),
$$

where we denote by 1 and $\epsilon$ the trivial and sign $\mathbb{Z} / 2 \mathbb{Z}$-representations, respectively. Then

$$
(\lambda) \times(0) \otimes \bigwedge^{\ell} \mathfrak{h}=\operatorname{Ind}_{S_{n-\ell} \times S_{\ell} \times(\mathbb{Z} / 2 \mathbb{Z})^{n}}^{W\left(B_{n}\right)}\left(\left.(\lambda)\right|_{S_{n-\ell} \times S_{\ell}} \otimes 1^{\otimes(n-\ell)} \otimes \epsilon^{\otimes \ell}\right)
$$

If we vary $\ell$ it follows that

$$
(\lambda) \times(0) \otimes \bigwedge \mathfrak{h}=\sum_{\ell=0}^{n} \sum_{\mu, \nu} c_{\mu, \nu}^{\lambda}(\mu) \times\left(\nu^{t}\right),
$$

where the interior sum is over all partitions $\mu$ of $n-\ell$ and $\nu$ of $\ell$, and $c_{\mu, \nu}^{\lambda}$ is the Littlewood-Richardson coefficient.

Since $\lambda$ is a rectangular partition, it follows from the LittlewoodRichardson rule that if $\nu=\lambda \backslash \mu$, then $c_{\mu, \nu}^{\lambda} \neq 0$. Here we regard $\mu$ as a left justified decreasing partition sharing the same upper left corner as $\lambda$ and $\nu$ is the left justified decreasing partition obtained by rotating by $180^{\circ}$ the complement of $\mu$ in $\lambda$.

We claim that every bipartition $(\mu) \times\left(\nu^{t}\right)$ where $\nu=\lambda \backslash \mu$ is in $\mathcal{F}_{\text {cusp }}\left(B_{n}\right)$. Write $\mu$ in the form

$$
\mu=(\underbrace{0, \ldots, 0}_{i_{0}}, \underbrace{1, \ldots, 1}_{i_{1}}, \ldots, \underbrace{d, \ldots, d}_{i_{d}})
$$


where $i_{j} \geq 0$ and $i_{0}+i_{1}+\cdots+i_{d}=d+1$. Then $\nu^{t}=\left(b_{1}, b_{2}, \ldots, b_{d}\right)$, where

$$
b_{j}=i_{0}+i_{1}+\cdots+i_{j-1}
$$

The symbol of $(\mu) \times\left(\nu^{t}\right)$ is obtained by adding in order $0,1, \ldots, d$ to the entries of $\mu$ (for the first row of the symbol) and $0,1, \ldots, d-1$ to the entries of $\nu^{t}$ (for the second row). It follows that the first row of the resulting symbol is

$$
\begin{gathered}
0,1, \ldots, b_{1}-1, b_{1}+1, b_{1}+2, \ldots, b_{2}, b_{2}+2, b_{2}+3, \ldots, b_{3}+1, \ldots \\
b_{d}+(d-2), b_{d}+d, \ldots, 2 d
\end{gathered}
$$

while the second row is

$$
b_{1}, b_{2}+1, b_{3}+2, \ldots, b_{d}+(d-1)
$$

Clearly the two rows are disjoint and their union equals $\{0,1,2, \ldots, 2 d\}$. By the characterization of families in type $B_{n}$, these are precisely all the $W$-types in $\mathcal{F}_{\text {cusp }}\left(B_{n}\right)$.

Type $\mathbf{D}_{\mathbf{n}}$. Here $n=d^{2}$ and $\sigma=(\underbrace{d, \ldots, d}_{d}) \times(0)$. The proof is completely analogous to type $B_{n}$ and we skip the details.

For the exceptional cases, we compute directly using GAP, the decomposition of the tensor product $\sigma \otimes \wedge \mathfrak{h}$.

Type $\mathbf{G}_{\mathbf{2}}$. The relevant $\sigma$ are $\phi_{1,3}^{\prime}, \phi_{1,3}^{\prime \prime}$, and $\phi_{2,2}$. The wedge representations are (in order): $\phi_{1,0}, \phi_{2,1}$, and $\phi_{1,6}$. We find

$$
\begin{aligned}
& \phi_{1,3}^{\prime} \otimes \bigwedge \mathfrak{h}=\phi_{1,3}^{\prime \prime} \otimes \bigwedge \mathfrak{h}=\phi_{1,3}^{\prime}+\phi_{1,3}^{\prime \prime}+\phi_{2,2} \\
& \phi_{2,2} \otimes \bigwedge \mathfrak{h}=2 \phi_{2,2}+\phi_{2,1}+\phi_{1,3}^{\prime}+\phi_{1,3}^{\prime \prime} .
\end{aligned}
$$

It follows that $\mathcal{F}_{\text {cusp }}\left(G_{2}\right) \subseteq \Theta^{-1}(\mathbf{0})$. Notice that in fact $\phi_{2,2}$ is sufficient for this conclusion.

Type $\mathbf{F}_{4}$. The relevant $\sigma$ are $1_{2}, 1_{3}, 4_{1}, 6_{1}, 4_{3}$, and $4_{4}$. The wedge representations are (in order): $1_{1}, 4_{2}, 6_{2}, 4_{5}$, and $1_{4}$. We find

$$
1_{2} \otimes \bigwedge \mathfrak{h}=1_{3} \otimes \bigwedge \mathfrak{h}=1_{2}+4_{3}+6_{1}+4_{4}+1_{3}
$$




$$
\begin{aligned}
& 4_{1} \otimes \bigwedge \mathfrak{h}=2 \cdot 4_{1}+6_{2}+2 \cdot 16_{1}+12_{1}+6_{1} \\
& 6_{1} \otimes \bigwedge \mathfrak{h}=3 \cdot 6_{1}+2 \cdot 4_{3}+2 \cdot 4_{4}+2 \cdot 16_{1}+1_{2}+1_{3}+4_{1}+9_{2}+9_{3} ; \\
& 4_{3} \otimes \bigwedge \mathfrak{h}=4_{4} \otimes \bigwedge \mathfrak{h}=2 \cdot 4_{3}+2 \cdot 4_{4}+1_{2}+9_{2}+2 \cdot 61+16_{1}+1_{3}+9_{3} .
\end{aligned}
$$

Taking the union of all the irreducible representations that occur in these decompositions, it follows that $\mathcal{F}_{\text {cusp }}\left(F_{4}\right) \subseteq \Theta^{-1}(\mathbf{0})$.

Type $\mathbf{E}_{6}$. The relevant $\sigma$ is $10_{s}$. The wedge representations are (in order): $\phi_{1,0}, \phi_{6,1}, \phi_{15,5}, \phi_{20,10}, \phi_{15,17}, \phi_{6,25}$, and $\phi_{1,36}$, in Carter's notation [11]. We find

$$
10_{s} \otimes \bigwedge \mathfrak{h}=3 \cdot 10_{s}+4 \cdot 60_{s}+3 \cdot 90_{s}+20_{s}+80_{s}
$$

It follows that $\mathcal{F}_{\text {cusp }}\left(E_{6}\right) \subseteq \Theta^{-1}(\mathbf{0})$.

Type $\mathbf{E}_{\mathbf{8}}$. The relevant $\sigma$ are $168_{y}$ and $420_{y}$. The wedge representations are (in order): $\phi_{1,0}, \phi_{8,1}, \phi_{28,8}, \phi_{56,19}, \phi_{70,32}, \phi_{56,49}, \phi_{28,68}, \phi_{8,91}$, and $\phi_{1,120}$, in Carter's notation [11]. We find

$$
\begin{aligned}
168_{y} \otimes \bigwedge \mathfrak{h}= & 3 \cdot 168_{y}+4 \cdot 1344_{w}+3 \cdot 420_{y}+3 \cdot 1134_{y}+2 \cdot 3150_{y}+2 \cdot 448_{w} \\
& +2 \cdot 2016_{w}+2 \cdot 5600_{w}+70_{y}+1400_{y}+1680_{y}+2688_{y}+4200_{y} \\
420_{y} \otimes \bigwedge \mathfrak{h}= & 5 \cdot 420_{y}+6 \cdot 1344_{w}+4 \cdot 2016_{w}+3 \cdot 168_{y}+4 \cdot 1134_{y}+3 \cdot 2688_{y} \\
& +4 \cdot 3150_{y}+3 \cdot 4200_{y}+2 \cdot 448_{w}+4 \cdot 5600_{w}+2 \cdot 7168_{w}+70_{y} \\
& +1400_{y}+1680_{y}+4536_{y}+5670_{y} .
\end{aligned}
$$

It follows that $\mathcal{F}_{\text {cusp }}(W) \backslash\left\{4480_{y}\right\} \subseteq \Theta^{-1}(\mathbf{0})$.

For the opposite inclusions, suppose that a $W$-type $\tau$ is contained in $\Theta^{-1}(\mathbf{0})=\left(\zeta_{0,1}^{*}\right)^{-1}(\mathbf{0})$. In particular, by Proposition 2.3, we have

$$
\sigma\left(\Omega_{W, 1}\right)=-\left\langle\Omega_{\mathbf{H}_{0,1}}, \mathbf{0}\right\rangle=0
$$

where $\left\langle\Omega_{\mathbf{H}_{0,1}}, \mathbf{0}\right\rangle$ denote the natural evaluation pairing between $Z\left(\mathbf{H}_{0,1}\right)$ and Spec $Z\left(\mathbf{H}_{0,1}\right)=X_{1}(W)$. But then Theorem 4.3, implies that

$$
a(\mathcal{F})=a(\mathcal{F} \otimes \text { sgn }),
$$


where $\mathcal{F}$ is the family containing $\sigma$. For exceptional Weyl groups $G_{2}, F_{4}$, and $E_{6}$, one can immediately see that the only families that have this property are the cuspidal ones.

In $E_{8}, \mathcal{F}_{\text {cusp }}$ is not the only family with the property that $\mathcal{F}=\mathcal{F} \otimes$ sgn. The other family with this property is a singleton, $\left\{2100_{y}\right\}$. Hence, this argument implies that $\Theta^{-1}(\mathbf{0}) \subseteq \mathcal{F}_{\text {cusp }}\left(E_{8}\right) \cup\left\{2100_{y}\right\}$.

Remark 4.7. The method of one- $W$-type modules doesn't yield any direct results for type $E_{7}$. The last part of the proof of Theorem 4.6 applies however to $E_{7}$ too and it gives

$$
\left\{512_{a}^{\prime}\right\} \subseteq \Theta^{-1}(\mathbf{0}) \subseteq\left\{512_{a}^{\prime}, 512_{a}\right\}=\mathcal{F}_{\text {cusp }}\left(E_{7}\right)
$$

A known necessary condition for a Calogero-Moser cell to be a singleton $\{\sigma\}$ is that the the fake degree $f_{\sigma^{*}}(t)$ must divide the Poincaré polynomial $P_{W}(t)\left[6\right.$, Lemma 3.3]. One can verify that this is not the case for $512_{a}^{\prime}$ and therefore we must have $\mathcal{F}_{\text {cusp }}\left(E_{7}\right)=\Theta^{-1}(\mathbf{0})$.

One can try to apply the same trick to $E_{8}$. In this case $f_{4480_{y}}(t)$ does not divide $P_{E_{8}}(t)$, but $f_{2100_{y}}(t)$ does. Again by the $a$-value argument in the last part of the proof of Theorem 4.6, the consequence is that there are only three possibilities:

(1) $\Theta^{-1}(\mathbf{0})=\mathcal{F}_{\text {cusp }}\left(E_{8}\right)$ and $\left\{2100_{y}\right\}$ is a CM-cell;

(2) $\Theta^{-1}(\mathbf{0})=\mathcal{F}_{\text {cusp }}\left(E_{8}\right) \backslash\left\{4480_{y}\right\}$ and $\left\{4480_{y}, 2100_{y}\right\}$ is a CM-cell;

(3) $\Theta^{-1}(\mathbf{0})=\mathcal{F}_{\text {cusp }}\left(E_{8}\right) \cup\left\{2100_{y}\right\}$.

The expectation is that the first possibility is the correct one. Notice that its verification is now equivalent with proving that $\left\{2100_{y}\right\}$ is a singleton CM-cell.

Remark 4.8. When the root system is not simply-laced $\left(B_{n}, F_{4}, G_{2}\right)$, Theorem 3.5 together with the same method as above can be used to construct nontrivial (cuspidal) Calogero-Moser cells. For example, in the case of $F_{4}$, the fact that the $W$-type $4_{1}$ extends to a one- $W$-type $\mathbf{H}_{0, c}$-module for all parameters $c$ implies that for all parameters $c=\left(c_{\ell}, c_{s}\right)$, the cuspidal CM cell $\Theta^{-1}(\mathbf{0})$ contains the set

$$
\left\{4_{1}, 6_{1}, 6_{2}, 16_{1}, 12_{1}\right\}
$$


In fact, for all but finitely many lines in this two-dimensional parameter space (see Theorem 3.5 for the list of non-generic values of $\left.\left(c_{\ell}, c_{s}\right)\right), \Theta^{-1}(\mathbf{0})$ equals the set in (4.4.13). One can see that (4.4.13) matches in fact a Lusztig twosided cell as defined for the Hecke algebra with generic unequal parameters by Geck [16, Table 2].

\section{Acknowledgments}

I thank G. Bellamy and U. Thiel for useful discussions regarding this work and their paper [7]. I also thank the referee for several useful comments and corrections.

Most of the ideas in this paper and in my previous work on Hecke algebras have been crucially influenced by Lusztig's transformative contributions to the representation theory of finite and $p$-adic groups and affine Hecke algebras. The results of this paper were presented in a talk at the Taipei Conference in Representation Theory, in honor of G. Lusztig's 70th birthday, in January 2016. I am grateful to the organizers for the invitation to participate in this conference and to contribute to this volume in the honor of George Lusztig.

\section{References}

1 D. Alvis, Induce/restrict matrices for exceptional Weyl groups, arXiv:math/0506377, 2005 .

2 D. Barbasch, D. Ciubotaru and P. Trapa, Dirac cohomology for graded affine Hecke algebras, Acta Math., 209 (2012), no. 2, 197-227.

3 D. Barbasch and A. Moy, Classification of one $K$-type representations, Trans. Amer. Math. Soc., 351 (1999), no. 10, 4245-4261.

4 G. Bellamy, The Calogero-Moser partition for $G(m, d, n)$, Nagoya Math. J., 207 (2012), 47-77.

5 G. Bellamy, Cuspidal representations of rational Cherednik algebras at $t=0$, Math. Z., 269 (2011), no. 3-4, 609-627.

6 G. Bellamy, On singular Calogero-Moser spaces, Bull. Lond. Math. Soc., 41 (2009), no. $2,315-326$.

7 G. Bellamy and U. Thiel, Cuspidal Calogero-Moser and Lusztig families for Coxeter groups, J. Algebra, 462 (2016), 197-252. 
8 W. Beynon and G. Lusztig, Some numerical results on the characters of exceptional Weyl groups, Math. Proc. Cambridge Philos. Soc., 84 (1978), no. 3, 417-426.

9 C. Bonnafé and R. Rouquier, Calogero-Moser versus Kazhdan-Lusztig cells, Pacific J. Math., 261 (2013), no. 1, 45-52.

10 K.A. Brown, I. Gordon, Poisson orders, symplectic reflection algebras and representation theory, J. Reine Angew. Math., 559 (2003), 193-216

11 R. Carter, Finite groups of Lie type, Wiley Classics Library, John Wiley \& Sons, Ltd., Chichester, 1993.

12 D. Ciubotaru, Dirac cohomology for symplectic reflection algebras, Selecta Math. (N.S.) 22 (2016), no. 1, 111-144.

13 D. Ciubotaru, A. Moy, Dirac cohomology of one- $W$-type representations, Proc. Amer. Math. Soc., 143 (2015), 1001-1013.

14 P. Etingof, V. Ginzburg, Symplectic reflection algebras, Calogero-Moser space, and deformed Harish-Chandra homomorphism, Invent. Math., 147 (2002), 243-348.

15 Martin Schnert et.al., GAP - Groups, Algorithms, and Programming - version 3 release 4 patchlevel 4, Lehrstuhl D für Mathematik, Rheinisch Westfälische Technische Hochschule, Aachen, Germany, 1997.

16 M. Geck, Computing Kazhdan-Lusztig cells for unequal parameters, J. Algebra, 281 (2004), no. 1, 342-365.

17 M. Geck, G. Hiss, F. Lübeck, G. Malle and G. Pfeiffer, CHEVIE - A system for computing and processing generic character tables for finite groups of Lie type, Weyl groups and Hecke algebras, Appl. Algebra Engrg. Comm. Comput., 7 (1996), 75-210.

18 I. Gordon, Baby Verma modules for rational Cherednik algebras, Bull. London Math. Soc., 35 (2003), no. 3, 321-336.

19 I. Gordon and M. Martino, Calogero-Moser space, restricted rational Cherednik algebras and two-sided cells, Math. Res. Lett., 16 (2009), no. 2, 255-262.

20 G. Lusztig, Characters of reductive groups over a finite field, Annals of Mathematics Studies, 107, Princeton University Press, Princeton, NJ, 1984.

21 G. Lusztig, Affine Hecke algebras and their graded version, J. Amer. Math. Soc., 2 (1989), no. 3, 599-635.

22 M. Martino, Blocks of restricted rational Cherednik algebras for $G(m, d, n)$, J. Algebra, 397 (2014), 209-224.

23 E. Opdam, A remark on the irreducible characters and fake degrees of finite real reflection groups, Invent. Math., 120 (1995), no. 3, 447-454.

24 R. Rouquier, Familles et blocs d'algèbres de Hecke, C. R. Acad. Sci. Paris Sér. I Math., 329 (1999), no. 12, 1037-1042. 